\title{
Geomagnetically Quiet Period Analysis of Relativistic Electrons, Auroral Precipitation, Joule Heating, and Ring Current During the Years of 1999, 2000 and 2004
}

\author{
R. K. Mishra, A. Gautam, P. Poudel, N. Parajuli, A. Silwal, \\ B. Adhikari, B. R. Tiwari and S. P. Gautam
}

Journal of Nepal Physical Society

Volume 7, Issue 2, June 2021

ISSN: 2392-473X (Print), 2738-9537 (Online)

Editors:

Dr. Binod Adhikari

Dr. Bhawani Joshi

Dr. Manoj Kumar Yadav

Dr. Krishna Rai

Dr. Rajendra Prasad Adhikari

Mr. Kiran Pudasainee

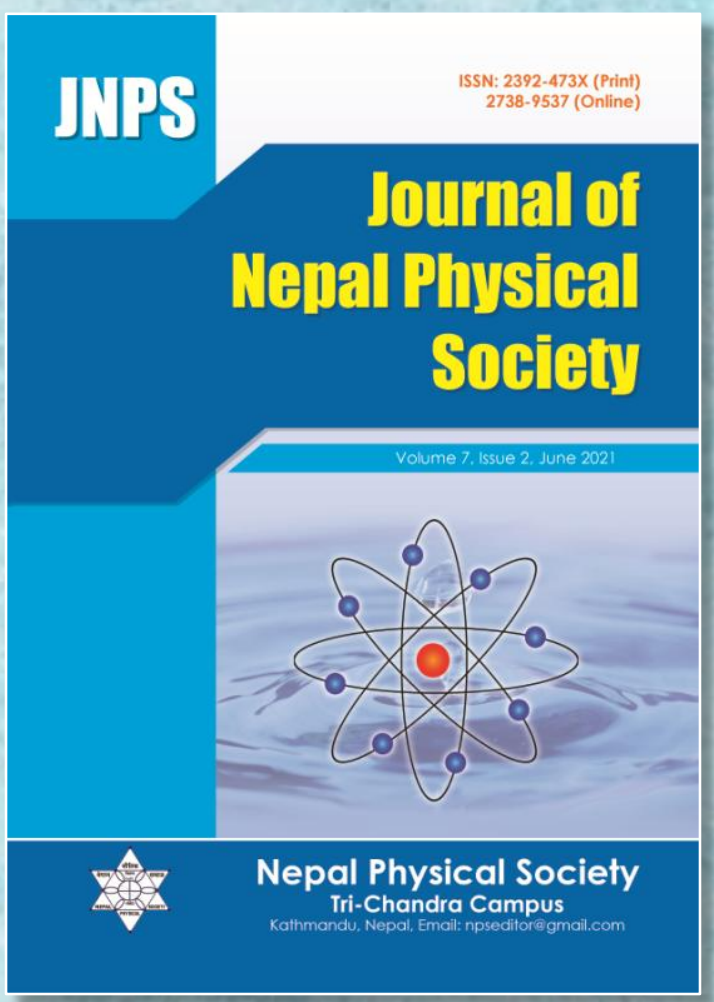

JNPS, 7 (2), 126-137 (2021)

DOI: https://doi.org/10.3126/jnphyssoc.v7i2.38633

Published by:

Nepal Physical Society

P.O. Box: 2934

Tri-Chandra Campus

Kathmandu, Nepal

Email: nps.editor@gmail.com 


\title{
Geomagnetically Quiet Period Analysis of Relativistic Electrons, Auroral Precipitation, Joule Heating, and Ring Current During the Years of 1999, 2000 and 2004
}

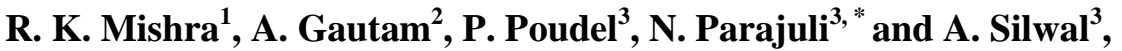 \\ B. Adhikari ${ }^{1,3}$, B. R. Tiwari ${ }^{1}$, S. P. Gautam ${ }^{4}$ \\ ${ }^{1}$ Department of Physics, St. Xavier's College, Maitighar, Kathmandu, Nepal \\ ${ }^{2}$ Department of Physics, Birendra Multiple Campus, Tribhuvan University, Bharatpur, Nepal \\ ${ }^{3}$ Department of Physics, Patan Multiple Campus, Tribhuvan University, Lalitpur, Nepal \\ ${ }^{4}$ Central Department of Physics, Tribhuvan University, Kirtipur, Nepal \\ "Corresponding Email: nikiparnami1@gmail.com
}

Received: 14 April, 2021; Revised: 13 May, 2021; Accepted: 22 June, 2021

\begin{abstract}
This work presents the study of the quietest time variation in relativistic electrons, auroral precipitation, ring current, and joule heating during 1999, 2000, and 2004. Geostationary Operational Environmental Satellite (GOES) data on relativistic electrons with energies above $0.6 \mathrm{MeV}, 2 \mathrm{MeV}$, and $4 \mathrm{MeV}$ were analyzed. The time-series analysis of the relativistic electrons over a 24-hour averaged interval reveals a precise 24-hour modulation of the relativistic electron population during all seasons for energies above $0.6 \mathrm{MeV}$ and $2 \mathrm{MeV}$, and during the winter season for higher energies above $4 \mathrm{MeV}$. In addition, relativistic electron fluxes at energies above $0.6 \mathrm{MeV}$ and above $2 \mathrm{MeV}$ were higher during the descending phase of the solar cycle compared to the ascending and solar-maximum phases. The cross-correlation analysis presented a strong correlation of Joule heating, ring current, and auroral precipitation with the relativistic electron population in three energy bands considered, as indicated by the zero-time lag. Studying the quiet time variation of relativistic electrons will lead to more complete ionospheric models, which were previously limited to the geomagnetically disturbed period.
\end{abstract}

Keywords: Auroral Precipitation, Relativistic Electron, Ring Current, Magnetosphere, Joule Heating, Cross-Correlation.

\section{INTRODUCTION}

Interplanetary structures with the Earth's magnetosphere-ionosphere system can produce geomagnetic storm, substorm, and high energy charged particles trapping in the radiation belt $[1,2]$. Hundreds of satellites in geosynchronous orbit near the radiation belt are vulnerable to relativistic electrons. These relativistic electrons in an energy range of $\mathrm{MeV}$ can cause damage to the satellite components or even loss of satellites in severe events [3, 4]. Thus, the prediction of the relativistic electron population, understanding their variation pattern, and study of their relationship with other interplanetary parameters are essential for the safety of satellites.
In the past few decades, considerable studies have been made to understand the origin and variability of the relativistic electrons in the radiation belt [58]. The relativistic electron flux at the outer zone of the magnetosphere is driven by high-speed solar wind streams [9], and the southern turning of the Interplanetary Magnetic Field (IMF) has a major contribution in driving the relativistic electron population [10]. The final relativistic electron population is first seeded by the substorms and is accelerated in the presence of high-speed solar wind $[11,12]$.

The electrons in the radiation belt exhibit seasonal, semi-annual, and solar cycle dependence on their time scales varying from a fraction of a second to 
several years. Numerous studies [13, 14] have identified seasonal and semi-annual variation, which can be explained in terms of an increased southward component of IMF in the geocentric solar magnetospheric coordinate system - geometric effect [15], the Earth's position in the heliosphere axial effect [16], and the angle made by the solar wind with the Earth's rotational axis - equinoctial effect [17]. Baker et al. [18] have shown that equinoctial fluxes are thrice as large as solstitial fluxes. They have suggested that seasonal variation of relativistic electron fluxes is due to the geometrical effect: Russell-Mcpherron (RM) effect and solar wind-guided instability related to the equinoctial effect. Later, Li et al. [13] observed that the peak time of electron flux lagged the nominal equinoxes and suggested that the equinoctial effect by itself could not interpret seasonal dependence of relativistic electron fluxes.

Radiation belts and ring currents constitute the major magnetospheric particle population. The drift of charged particles of the outer radiation belt around Earth forms the ring current. Large amounts of magnetospheric particles are trapped into the inner magnetosphere during storms and substorms, which increases the intensity of the ring current $[19,20]$. The quiet time ring current is chiefly conveyed by solar wind origin, whereas active processes are associated with an increment in $\mathrm{O}^{+}$ions concentration (ionospheric origin) $[21,22]$. When the solar wind enters the Earth's magnetosphere, it causes the precipitation of ions and electrons, causing the aurora. These auroral precipitations give rise to ionospheric currents, which drive the joule heating [23]. Joule heating arises from the friction between the neutral particles and the charged particles in the ionospheric currents [24]. The overall analysis of mentioned literature reveals the association of relativistic particle flux with Auroral precipitation, Joule heating, and ring current.

This work covers the annual and seasonal variations of the relativistic electron population of a different energy band in the outer radiation belt. Along with this, we study the correlation of relativistic electron flux with ring current, auroral precipitation, and joule heating on a seasonal basis: Winter (November to February), Spring (March, April), Summer (May to August), and Autumn (September, October). We believe that this type of study contributes to the model of the relativistic electron population in the radiation belt. This paper is structured as follows: Section 2 describes the data source and techniques used in the study.
Section 3 presents our work's findings, and the final section includes the conclusion based on the observed results.

\section{DATA SET AND METHODOLOGY}

In this work, the relativistic electron flux data of energy bands $>0.6 \mathrm{MeV},>2 \mathrm{MeV}$, and $>4 \mathrm{MeV}$ provided by the NOAA (National Oceanic and Atmospheric Administration) operated Global Positioning System (GOES) were used. GOES is equipped with a space environment to monitor instrument subsystem that continuously monitors energetic particles on the radiation belt near the geostationary orbit [25], the data from which can be accessed through the GOES data access link at https://www.ngdc.noaa.gov/stp/

satellite/goes/dataaccess.html. The observations are made throughout the years 1999, 2000, and 2004, which correspond to the ascending, maximum, and descending phases of solar cycle 23, respectively. Joule heating $(\mathrm{Uj})$, Auroral Precipitation energy $\left(U_{A}\right)$, and Ring Current energy $\left(U_{R}\right)$ are calculated using the procedure suggested by Ostgaard et al. $[26,27]$ :

$$
\begin{gathered}
U_{R}=4.10^{4}\left[\left(\frac{S Y M H_{t+1}-S Y M H_{t}}{60}\right)+\left(\frac{S Y M H}{4.60 .60}\right)\right] 10^{9} \\
U_{J}=(0.54 A E+1.8) 10^{9} \ldots(2) \\
U_{A}=(4.4 \sqrt{|A L|}-7.6) 10^{9} \ldots(3)
\end{gathered}
$$

AL is the westward auroral electrojet, called amplitude lower index, AE is the auroral electrojet index, and SYM-H is the symmetric horizontal component of the geomagnetic field. The data for $\mathrm{AE}, \mathrm{AL}$, and SYM-H are derived from OMNI (Operating Mission as Nodes on the Internet), which provides interplanetary magnetic field and plasma parameter data from different geocentric orbiting satellites through its site link: https://www.omniweb.gsfc.nasa.gov.

\section{Cross-Correlation Analysis}

Cross-correlation is a statistical technique used to compare multiple time-series data to determine how well they relate to each other and at what time they best line up [28-30]. The association between the compared parameters is measured in the index, called the cross-correlation coefficient, ranging from -1 to +1 . The coefficient value around zero reveals 
poor relation among the compared parameters, whereas \pm 1 manifests a high correlation $[31,32]$. The plot of cross-correlation coefficient versus time helps to visualize to what extent the comparable indices lead or lag each other [33]. We have implied a crosscorrelation approach between the relativistic electron flux of different energy bands and Joule heating, auroral precipitation, and ring current to evaluate the heating and electrical system of Earth's upper atmosphere the variation of energetic electrons population on Earth's radiation belt.

\section{RESULTS}

The results for the seasonal and yearly variation of relativistic electrons in the three energy bands above $0.6 \mathrm{MeV}$, above $2 \mathrm{MeV}$, and above $4 \mathrm{MeV}$ for the years 1999, 2000, and 2004 during solar cycle 23 are presented in the following sections. The three energy bands are referred to as RE $>0.6$ $\mathrm{MeV}, \mathrm{RE}>2 \mathrm{MeV}$ and $\mathrm{RE}>4 \mathrm{MeV}$, respectively from here on. The cross-correlation of the relativistic electron population with Joule heating, ring current, and auroral precipitation are also presented for the three energy bands for the three years considered.

\subsection{Seasonal Variation of Relativistic Electron}

Figure 1 shows the seasonal averages of the relativistic electrons in the three energy bands for the year 1999. For the energy bands $\mathrm{RE}>0.6 \mathrm{MeV}$ and $\mathrm{RE}>2 \mathrm{MeV}$, we noticed that the variation of autumn fluxes over the 24 hours was the greatest. The variations of spring and winter followed a similar trend in phase and amplitude over the 24 hours. The amplitudes of spring and winter fluxes were generally the least; however, the summer and autumn fluxes had greater amplitudes. The variation of summer flux was smaller than that of autumn flux as well. Clearly, for the energies RE > $0.6 \mathrm{MeV}$ and $\mathrm{RE}>2 \mathrm{MeV}$, the autumn fluxes were more significant and more variable during 1999. For the high energies $\mathrm{RE}>4 \mathrm{MeV}$, the winter fluxes over a given 24-hour period were much greater than the other three seasonal fluxes on average.
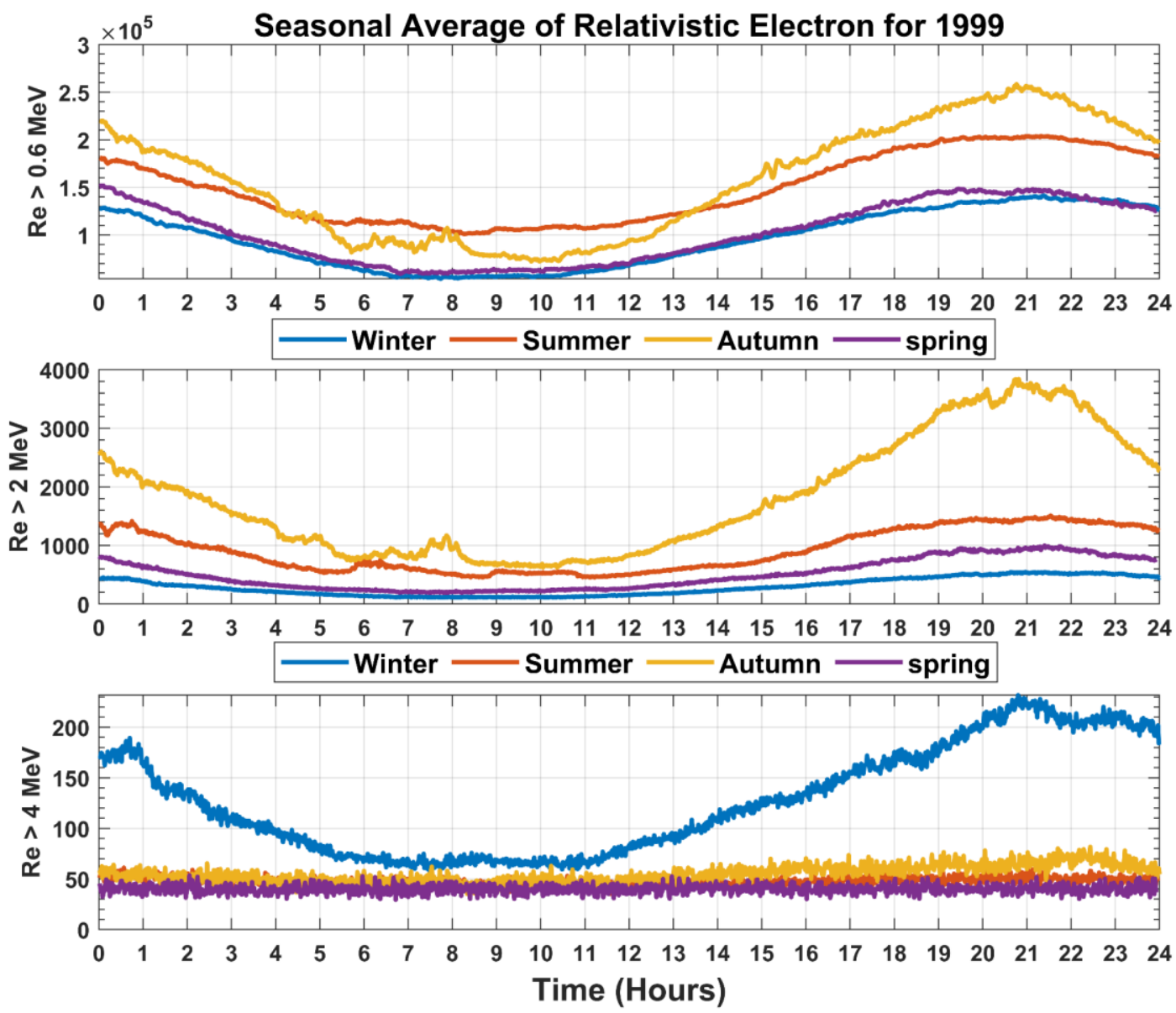

Fig. 1: Seasonal average fluxes of relativistic electrons for summer, winter, spring, and autumn during the year 1999. The fluxes are shown for three energy bands over 24 hours shown along the $x$-axis. 
The 24-hour average trend for all four seasons showed a trend such that the electron fluxes declined as the morning (time $=6$ UTC) approached, reaching the minimum during the early morning (6 UTC to 9 UTC) steadily climbed during the afternoon and evening. The fluxes reached a maximum during the time $=21$ UTC during the night and then decreased. The 24-hour modulation of the average fluxes was precise for the energy fluxes $\mathrm{RE}>0.6 \mathrm{MeV}$ and $\mathrm{RE}>2 \mathrm{MeV}$. The winter fluxes showed the high energy flux $\mathrm{RE}>4 \mathrm{MeV}$ trend, but the amplitudes were smaller for the other three seasons.

Similarly, figure 2 shows the seasonal averages for the relativistic electron fluxes over 24 hours during the year 2000. For the energy fluxes, RE $>0.6 \mathrm{MeV}$ and $\mathrm{RE}>2 \mathrm{MeV}$, the summer and spring fluxes were the smallest with the slightest variation. However, the winter fluxes were the greatest for the above energies during 2000, unlike 1999. The autumn fluxes for energies $\mathrm{RE}>0.6 \mathrm{MeV}$ and $\mathrm{RE}$ $>2 \mathrm{MeV}$ showed less variation during the year 2000 compared to the year 1999.
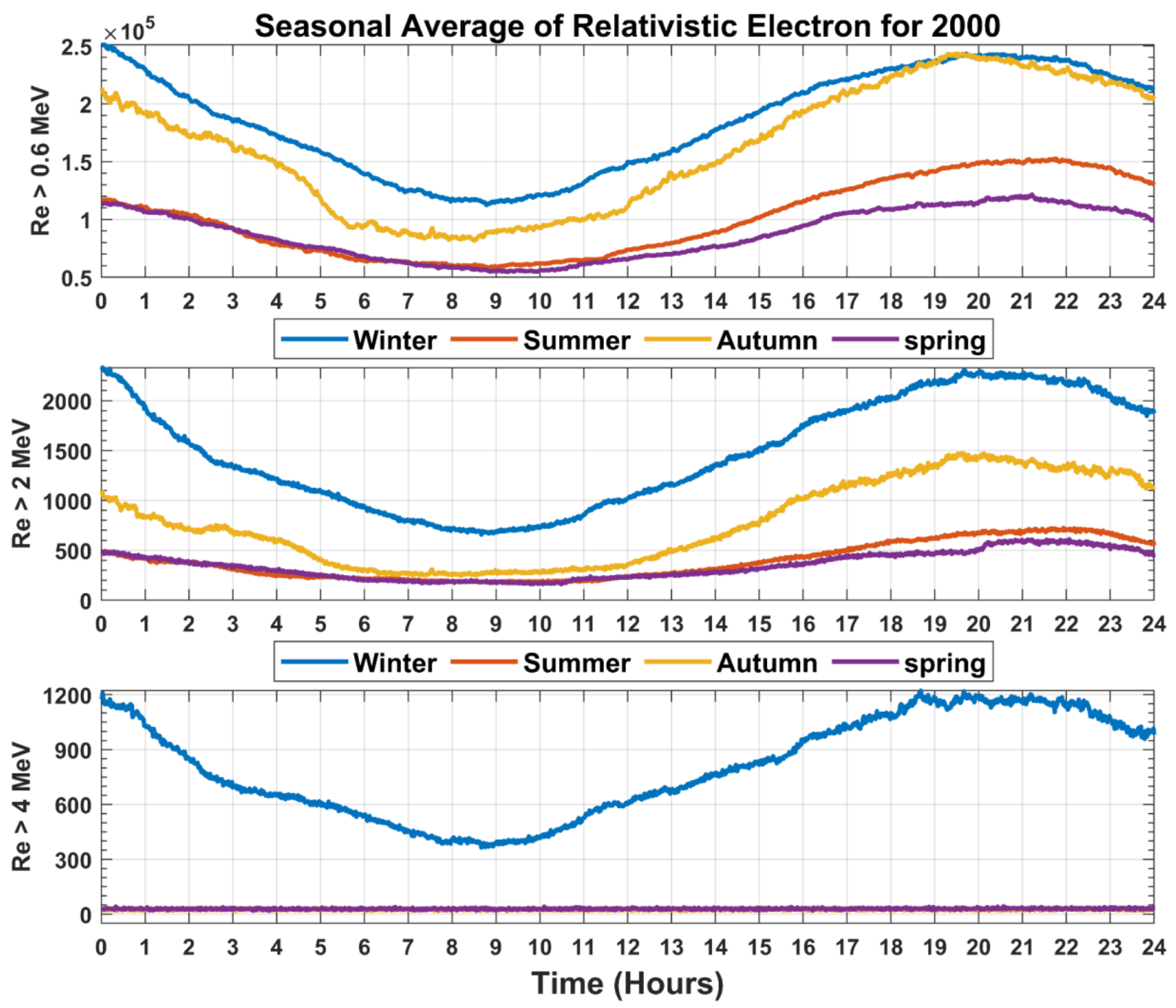

Fig. 2: Seasonal average fluxes of relativistic electrons for summer, winter, spring, and autumn during the year 2000. The fluxes are shown for three energy bands over 24 hours, shown along the x-axis.

The winter fluxes for the higher energies $\mathrm{RE}>4$ $\mathrm{MeV}$ were much greater than the other three seasons. The 24-hour modulation was clear for the winter high energy fluxes, but the other three seasonal fluxes did not exhibit any clear modulation. For the energy fluxes $\mathrm{RE}>0.6 \mathrm{MeV}$ and $\mathrm{RE}>2 \mathrm{MeV}$, the 24-hour modulation can be confirmed for the four seasons from figure 2 . Figure 3 shows the seasonal 
averages of the relativistic electron fluxes over 24 hours for the year 2004. For the energies RE $>0.6$ $\mathrm{MeV}$ and $\mathrm{RE}>2 \mathrm{MeV}$, the winter fluxes were the greatest, with the other three seasonal fluxes comparable. The winter flux remained considerably larger for the higher energies $\mathrm{RE}>4 \mathrm{MeV}$ than the other three fluxes. The 24-hour modulation of the relativistic electrons was clear for the lower energies for all four seasons and the winter season at higher energies.
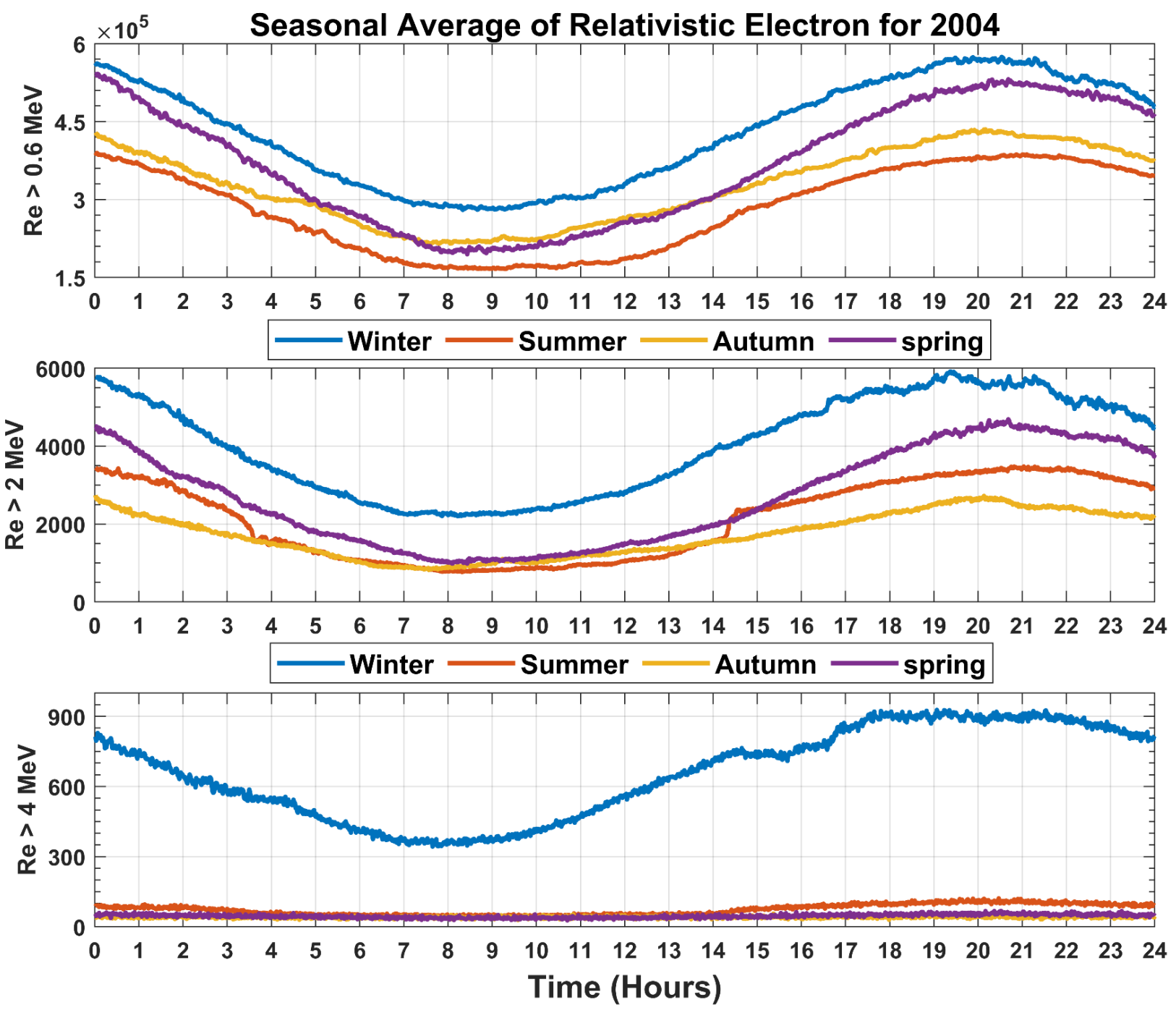

Fig. 3: Seasonal average fluxes of relativistic electrons for summer, winter, spring, and autumn during the year 2004. The fluxes are shown for three energy bands over 24 hours shown along the $x$-axis.

From the seasonal variation for 1999, 2000, and 2004 during solar cycle 23, it was clear that the relativistic electron population consisted mainly of lower energy electrons at $\mathrm{RE}>0.6 \mathrm{MeV}$ and $\mathrm{RE}>2 \mathrm{MeV}$. The populations of electrons at RE $>0.6 \mathrm{MeV}$ and $\mathrm{RE}>2 \mathrm{MeV}$ were of the order of $2 \times 10^{5}$ And 3000 , respectively. The population of higher energy electrons at RE $>4 \mathrm{MeV}$ was of the order 200 to 1400 only. In addition, the 24hour modulation was readily apparent for the relativistic electrons for lower energies during all four seasons, whereas for the higher energy electrons, the modulation was less apparent for the seasons other than winter.

\subsection{Yearly Variation of Relativistic Electron}

Figure 4 shows the yearly averaged variation of relativistic electrons over the 24 hours during the years 1999, 2000, and 2004 of the solar cycle 23. The panels of the figure show the variation for the three energy bands RE $>0.6 \mathrm{MeV}, \mathrm{RE}>2 \mathrm{MeV}$ and $\mathrm{RE}>4 \mathrm{MeV}$. 

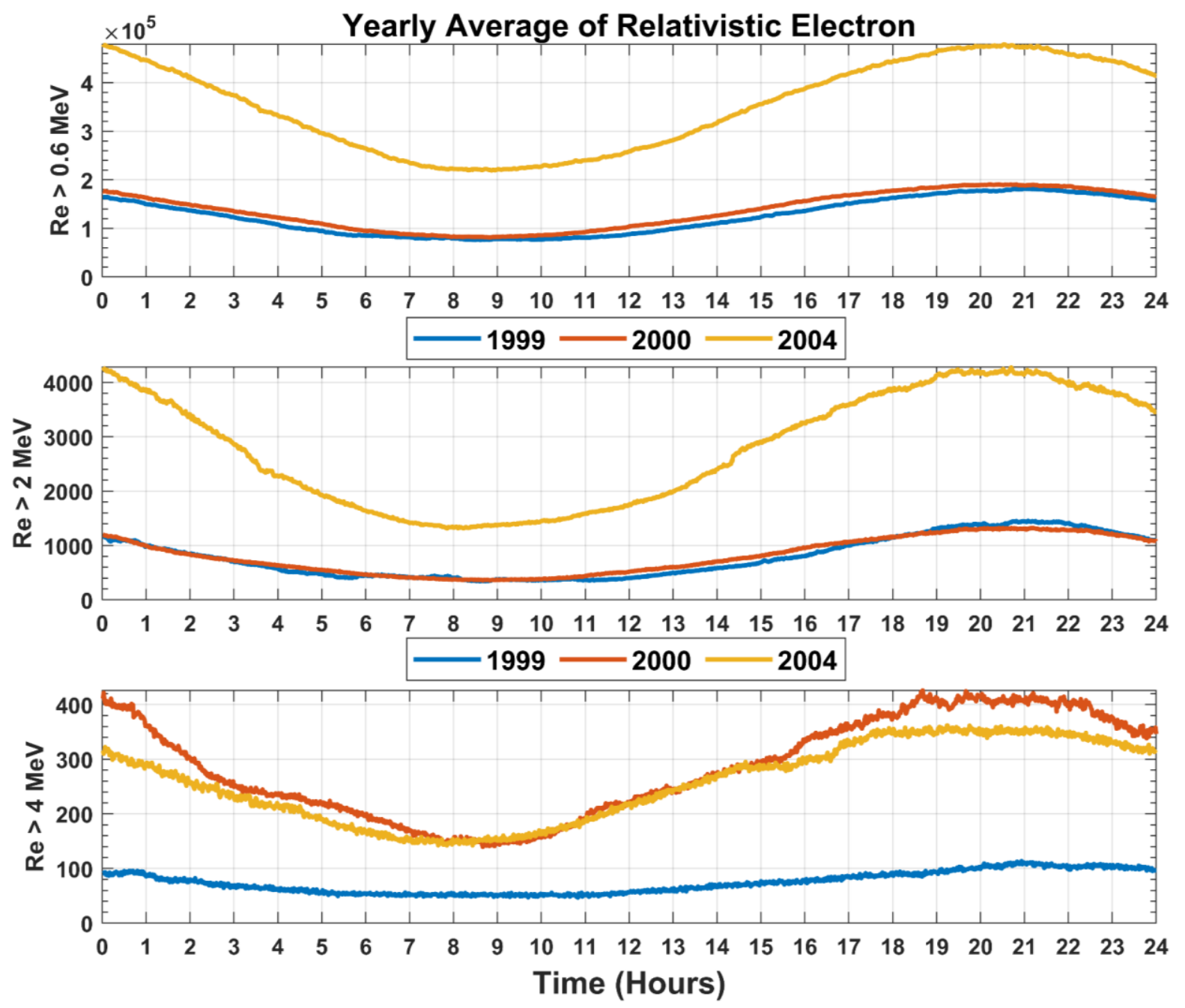

Fig. 4: Yearly averaged variation of relativistic electrons in the years 1999, 2000, and 2004 during solar cycle 23 for three energy bands during a 24-hour time.

Again, the yearly variation of relativistic electrons confirms the 24-hour modulation for all three energy bands during all three solar cycle phases. The average relativistic electron populations range from about $1 \times 10^{5}$ to $2 \times 10^{5}$ for the energy band $\mathrm{RE}>0.6 \mathrm{MeV}$ during the years 1999 and 2000. The average relativistic electron population ranges from 400 to 1200 for the energy band $\mathrm{RE}>2 \mathrm{MeV}$ during the years 1999 and 2000. But for the year 2004 , the relativistic electron fluxes were greater for both the energy bands reaching up to $5 \times 10^{5}$ and 4000, respectively. Clearly, the years 1999, 2000, and 2004 were in the trend of increasing fluxes of high energy electrons $\mathrm{RE}>0.6 \mathrm{MeV}$ and $\mathrm{RE}>2 \mathrm{MeV}$. For the higher energy electrons RE > $4 \mathrm{MeV}$, the fluxes were in the range of 150 to 400 during the years 2000 and 2004. For the year 1999, the electron flux at $\mathrm{RE}>4 \mathrm{MeV}$ was smaller, reaching up to 110 .

The descending phase of the solar cycle, i.e., the year 2004, has higher relativistic electrons at RE > 0.6 $\mathrm{MeV}$ and $\mathrm{RE}>2 \mathrm{MeV}$ compared to the ascending (year 1999) and maximum (year 2000) phases. But for the relativistic electrons $\mathrm{RE}>4 \mathrm{MeV}$, the maximum phase (year 2000) and descending phase (2004) both have comparable electron fluxes.

\subsection{Yearly Variation of Auroral Precipitation, Ring Current, and Joule Heating}

Figure 5 shows the yearly variation of solar wind energy parameters, specifically the auroral precipitation $\left(\mathrm{U}_{\mathrm{A}}(\mathrm{W})\right)$, joule heating $\left(\mathrm{U}_{\mathrm{J}}(\mathrm{W})\right)$, and ring current $\left(\mathrm{U}_{\mathrm{R}}(\mathrm{W})\right)$ on quiet solar days over the years 1999, 2000 and 2004, respectively, during the solar cycle 23. 

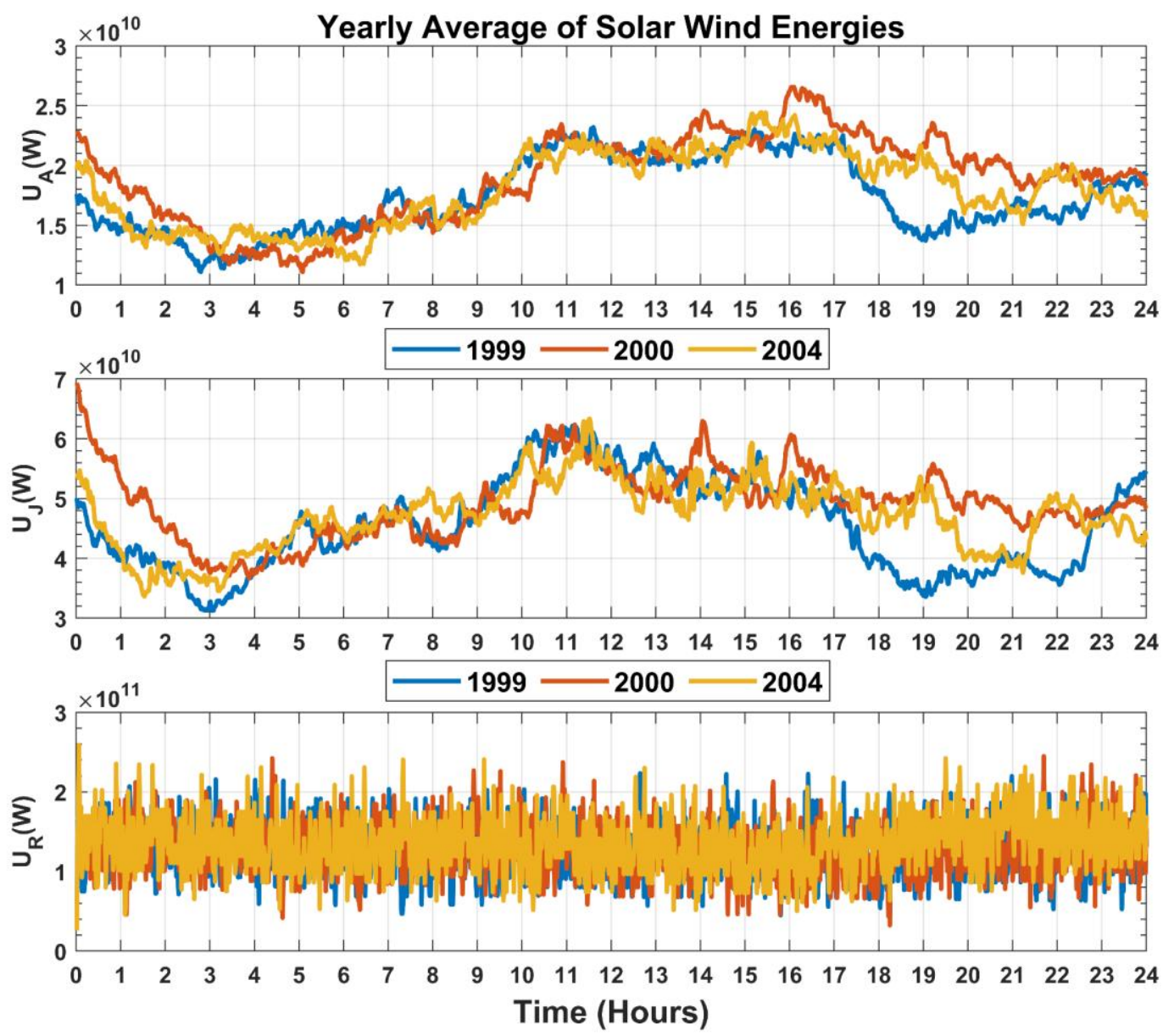

Fig. 5: Yearly variation of quiet day auroral precipitation, joule heating, and ring current over 1999, 2000 , and 2004.

Figure 5 clearly shows the auroral precipitation followed a 24-hour modulation like the relativistic electrons but with a different phase, and the short time variations were more numerous. The auroral precipitation decreased to a minimum just before the morning at time $=6$ UTC, then climbed steadily to a maximum at time $=15$ UTC, then started to decrease during the night. The modulation followed a similar pattern for the years 2000 and 2004, whereas, for the year 1999, the modulation was a bit different: the auroral precipitation rose earlier from the minimum during the morning, stayed longer during the maxima phase, and reached the next minima before midnight. The fluxes then started climbing during the night, reached a maximum, and declined until the morning. The period of modulation of fluxes for the year 1999 appeared to be shorter than 24 hours.

From figure 5, it is noticed that the modulation was less apparent for the ring current compared to relativistic electrons and auroral precipitation. The amplitude of the ring current also remained similar for the three years 1999, 2000, and 2004. The 24 hours averaged ring current remained mostly between $1 \times 10^{5}$ and $2 \times 10^{5}$ during the three years considered in the solar cycle 23 . Similarly, Joule heating was observed to fall to a minimum at time $=3$ UTC before the morning, steadily rising during the early afternoon and remaining high till the evening before dipping during the evening. The Joule heating then rose during midnight to complete the modulation before the early morning minima the next day.

\subsection{Cross-Correlation Analysis}

Figure 6 shows the cross-correlation analysis of relativistic electron fluxes with the three parameters: auroral precipitation $\left(\mathrm{U}_{\mathrm{A}}\right)$, Joule heating $\left(U_{J}\right)$, and ring current $\left(U_{R}\right)$ during the year 1999 in the three subpanels. The cross-correlation analysis is shown for the three energy bands RE > $0.6 \mathrm{MeV}, \mathrm{RE}>2 \mathrm{MeV}$ and $\mathrm{RE}>4 \mathrm{MeV}$. 


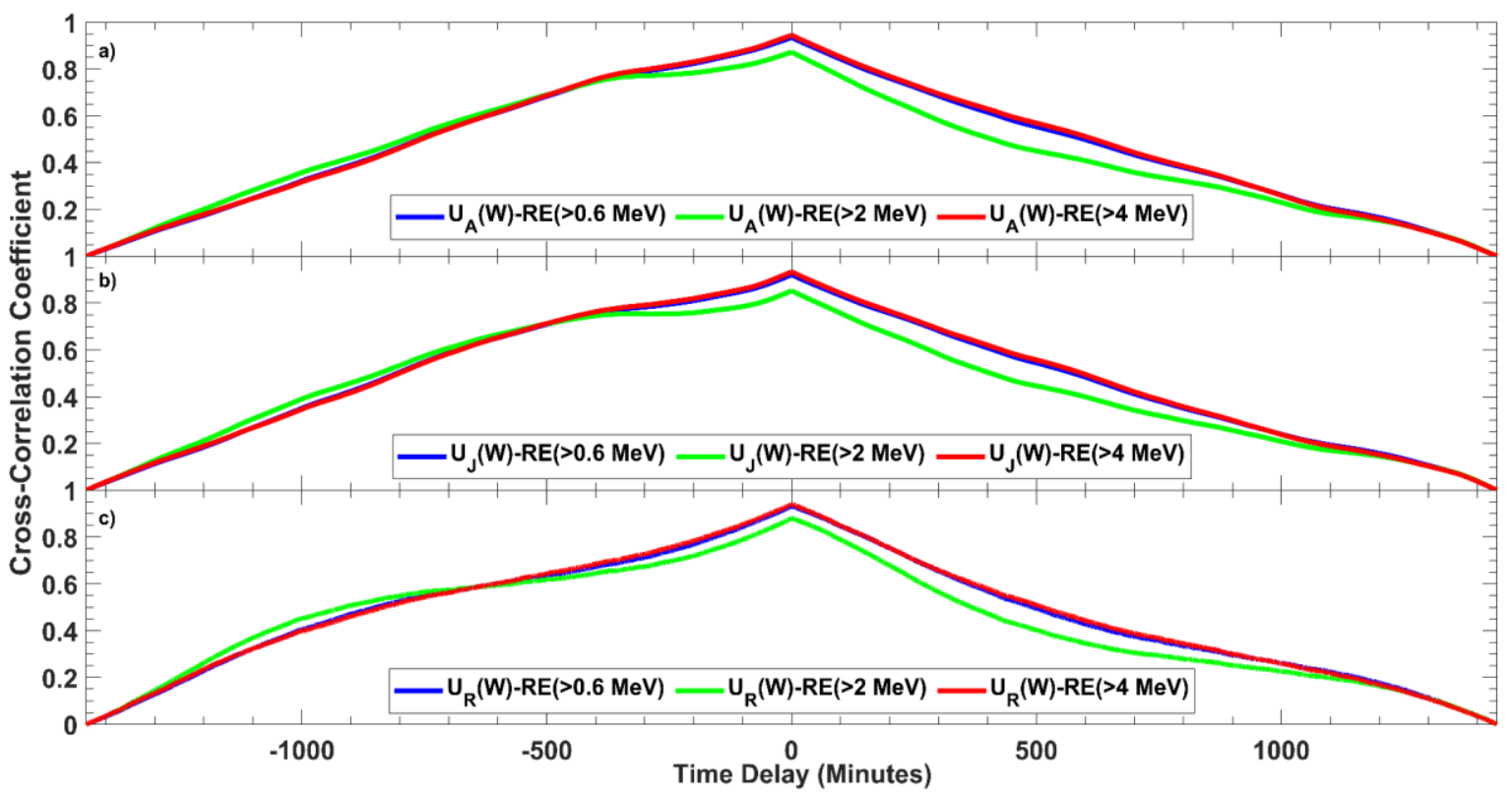

Fig. 6: Cross-correlation of auroral precipitation, joule heating, and ring current (subpanels: top to bottom) with relativistic electrons for the three energy bands $R E>0.6 \mathrm{MeV}, \mathrm{RE}>2 \mathrm{MeV}$, and $\mathrm{RE}>4 \mathrm{MeV}$ in the year 1999.

Figure 7 shows the cross-correlation analysis of relativistic electron fluxes with the three parameters: auroral precipitation $\left(\mathrm{U}_{\mathrm{A}}\right)$, Joule heating $\left(\mathrm{U}_{\mathrm{J}}\right)$, and ring current $\left(\mathrm{U}_{\mathrm{R}}\right)$ during the year 2000 in the three subpanels. The crosscorrelation analysis is shown for the three energy bands $\mathrm{RE}>0.6 \mathrm{MeV}, \mathrm{RE}>2 \mathrm{MeV}$ and $\mathrm{RE}>4$ $\mathrm{MeV}$.

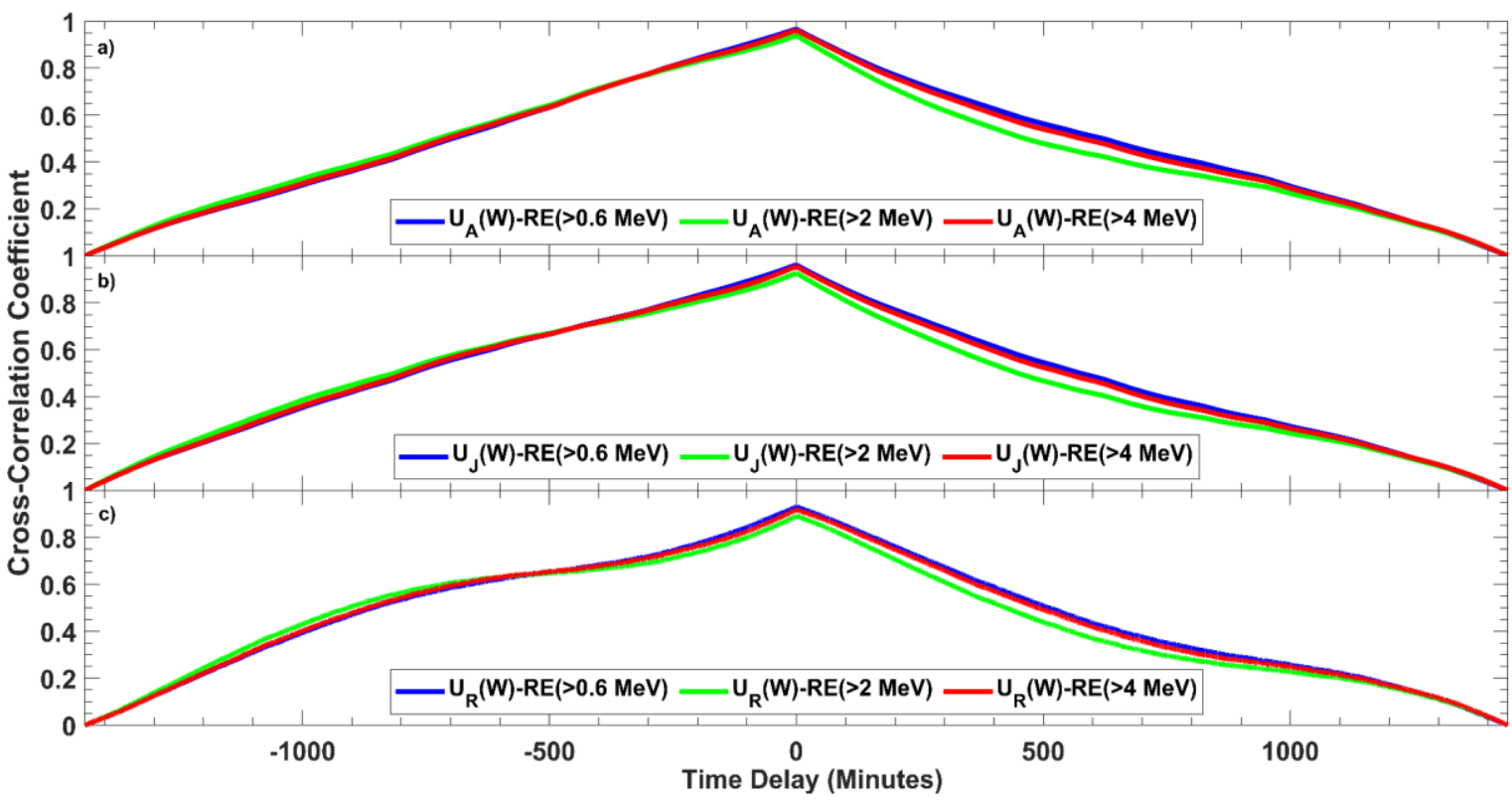

Fig. 7: Cross-correlation of auroral precipitation, joule heating, and ring current (subpanels: top to bottom) with relativistic electrons for the three energy bands $\mathrm{RE}>0.6 \mathrm{MeV}, \mathrm{RE}>2 \mathrm{MeV}$, and RE > $4 \mathrm{MeV}$ in the year 2000.

Figure 8 shows the cross-correlation analysis of relativistic electron fluxes with the three parameters: auroral precipitation $\left(\mathrm{U}_{\mathrm{A}}\right)$, Joule heating $\left(\mathrm{U}_{\mathrm{J}}\right)$, and ring current $\left(\mathrm{U}_{\mathrm{R}}\right)$ during the year 2004 in the three subpanels. The crosscorrelation analysis is shown for the three energy bands $\mathrm{RE}>0.6 \mathrm{MeV}, \mathrm{RE}>2 \mathrm{MeV}$ and $\mathrm{RE}>4$ $\mathrm{MeV}$. 


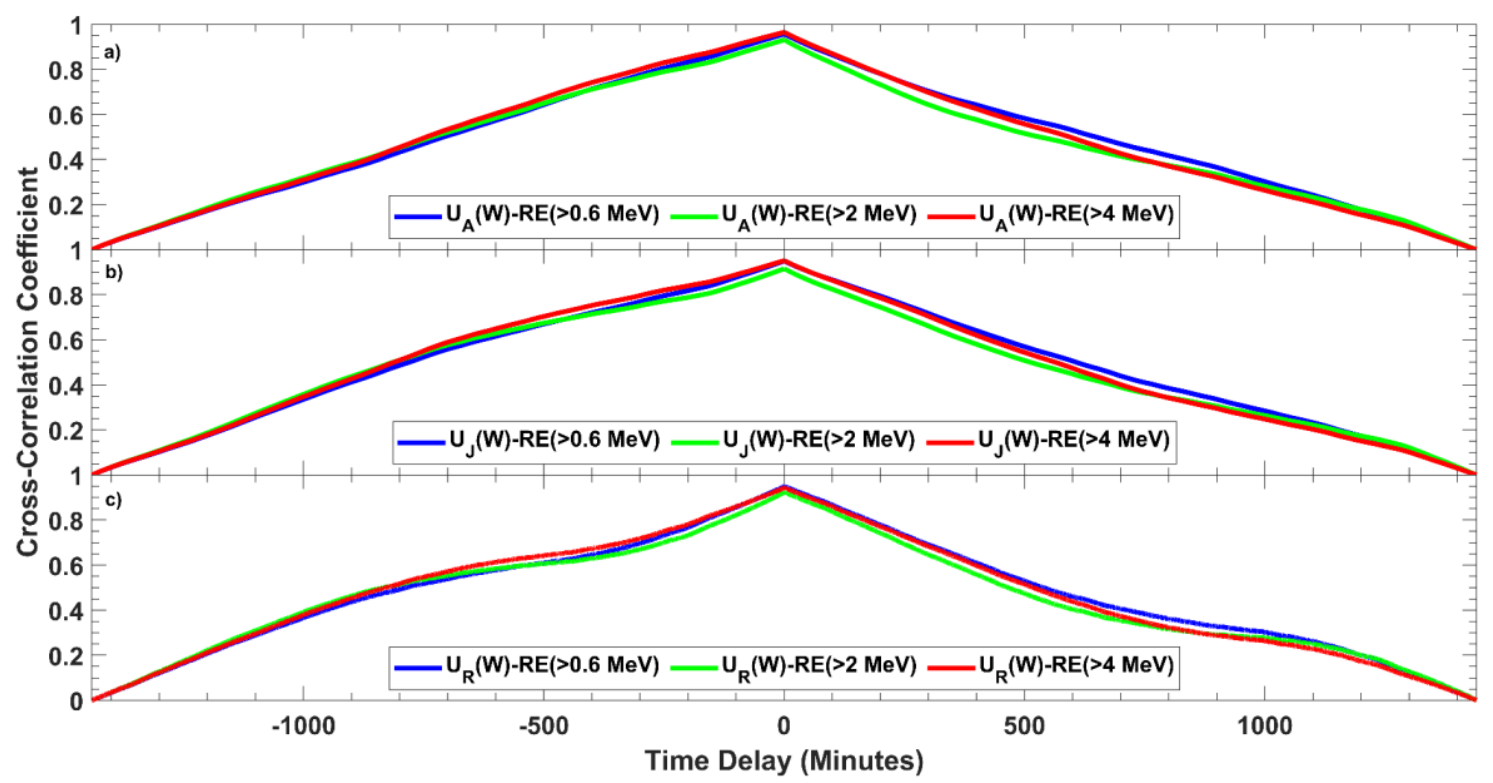

Fig. 8: Cross-correlation of auroral precipitation, Joule heating, and ring current (subpanels: top to bottom) with relativistic electrons for the three energy bands RE > 0.6 MeV, RE > $2 \mathrm{MeV}$, and RE > $4 \mathrm{MeV}$ in the year 2004.

The correlation of relativistic electrons and auroral precipitation was demonstrated from the crosscorrelation analysis for all three years 1999, 2000, and 2004. The maximum correlation around $\mathrm{r}=$ 0.95 was reached for all three years at nearly the zero-time lag. The auroral precipitation and relativistic electrons followed a synchronized modulation, and the correlation was very high for all three years. For all the three years and three energy bands, the correlation rose steadily from the negative time lag and reached the maximum at zero-time lag and steadily decreased over the positive time lag.

The cross-correlation analysis of ring current with relativistic electrons revealed that the ring current reached maximum correlation at zero-time lag similar to the auroral precipitation for all three energy bands in the three years 1999, 2000, and 2004 considered. Similarly, the Joule heating correlates with relativistic electron fluxes for all the three energy bands at zero-time lag. This is true for all the three years 1999, 2000 and 2004 considered. The maximum correlation of around 0.95 is reached for the time lag of 0 minutes in all three energy bands.

\subsection{Discussion}

From the above cross-correlation analyses, it is clear that the relativistic electron populations in all the three energy bands are highly correlated with Joule heating, ring current, and auroral precipitation during the three years 1999, 2000, and 2004. Specifically, the variation in all three factors and relativistic electron fluxes are in synchronization. Poudel et al. [34] had performed a cross-correlation analysis of relativistic electron flux with sunspot number, solar flux, and solar wind parameters. This work extends the cross-correlation to Joule heating, ring current, and auroral precipitation and, as explained above, finds high correlations with relativistic electron population. Poudel et al. [34] found a good correlation of relativistic electrons with solar wind velocity and plasma density. The correlation with Joule heating, ring current, and auroral precipitation further supports the inference because the plasma density in the ionosphere is closely related to the magnitude of Joule heating, ring current, and auroral precipitation. High-speed solar wind with high plasma density has been shown to affect the spatial structure of the radiation belts, driving up relativistic electrons in these regions [35]. Similarly, ring current is known to be enhanced during magnetic storms with greater plasma densities and high-speed solar winds [23]. When the solar wind enters the Earth's magnetosphere, the density of relativistic electrons increases in the initial phase, these electrons are then lost by different processes such as charge exchange of magnetospheric ions and pitch angle loss at mirror points in the radiation belts [36]. The precipitation of ions and electrons from the magnetosphere onto the ionosphere causes the aurora through collisions of these ions and electrons with neutral atoms in the atmosphere. These auroral precipitations also give rise to ionospheric currents, 
which drive the Joule heating of the atmosphere [23]. Joule heating is known to arise from the friction between the neutral particles and the charged particles in the ionospheric currents. Joule heating, therefore, acts as an energy sink for the energy deposited by the solar wind [24]. Previous studies [36] have studied auroral precipitation and Joule heating during geo-magnetically active periods, but this work studied them during the quiet periods. These energy deposition mechanisms can dramatically affect the state of the terrestrial atmosphere through the increase in neutral gas density, temperature, and ionization. Therefore, it is essential to take them into account in ionospheric modeling during magnetic storms and quiet periods. Ionospheric modeling is known to be important for real-world applications such as satellite trajectories and radio wave propagation [36].

From the time series analysis of the relativistic electron population, the 24-hour modulation of the relativistic electron population is clear for the energies $\mathrm{RE}>0.6 \mathrm{MeV}$ and $\mathrm{RE}>2 \mathrm{MeV}$ during all four seasons in the three years considered. But for the higher energies $\mathrm{RE}>4 \mathrm{MeV}$, the modulation is clear for winter only. This may be related to the much higher fluxes observed for winter than the other three seasons. Moreover, auroral precipitation and Joule heating clearly showed the 24-hour modulation, but no similar modulation of ring current was readily apparent. The 24-hour modulation and the relativistic electron fluxes seen during the geomagnetically quietest periods stress the importance of studying short-period variations of the relativistic electrons and associated energy parameters for accurate magnetospheric and ionospheric modeling. It is possible the Joule heating seen is associated with the partial ring currents present even during geomagnetically quiet days at all Dst index levels, as shown by Le et al. [37]. The relativistic electron fluxes seen could result from viscous interactions that transfer energy from the solar wind to the magnetic field lines, which can energize the electrons in the radiation belts [36].

In addition, Yeeram [38] had found that repetitive substorm activity driven by high-speed solar winds was related to relativistic electron enhancements during the descending phase of the solar cycle. In our work, we found the auroral precipitation, Joule heating, and ring current to be highly correlated during all the three phases of the solar cycle: ascending (1999), max (2000), and descending (2004). Adhikari et al. [39] had studied the seasonal variation of the magnetic field during the descending phase of the solar cycle. Future analyses can analyze the relationship between the interplanetary parameters and Joule heating, ring current, and auroral precipitation.

\section{CONCLUSION}

In this work, the relationship between relativistic electron population and three parameters, namely, auroral precipitation, ring current, and Joule heating, was studied for three years 1999, 2000, and 2004 of the solar cycle 23. Three energy bands of energies above $0.6 \mathrm{MeV}$, above $2 \mathrm{MeV}$, and greater than $4 \mathrm{MeV}$ were taken. The seasonal and yearly variations of the relativistic electron population in the three energy bands were studied using time series analysis over a 24-hour averaged interval. We studied the association of electron fluxes with auroral precipitation, Joule heating, and ring current through cross-correlation analysis. Based on the above analyses, we report the following findings:

1. A 24-hour modulation of the relativistic electron fluxes was reported for the energy bands above $0.6 \mathrm{MeV}$ and above $2 \mathrm{MeV}$ for all four seasons. This modulation was present over the three phases of the solar cycle in 1999, 2000, and 2004. For the higher energies above $4 \mathrm{MeV}$, the modulation was clear only during winter.

2. A 24-hour modulation of the Joule heating and auroral precipitation was also observed, but no modulation was readily apparent for the ring current. The modulation was present during all the three years 1999, 2000 and 2004.

3. The generation and loss of the relativistic electrons on a 24-hour interval in the absence of magnetic storms warrant further investigation for the underlying physical mechanisms.

4. Relativistic electron fluxes at energies above 0.6 $\mathrm{MeV}$ and above $2 \mathrm{MeV}$ were higher during the descending phase of the solar cycle (the year 2004) compared to the ascending phase (the year 1999) and maximum solar phase (the year 2000).

5. The cross-correlation analysis revealed a strong correlation $(r>0.9)$ of Joule heating, ring current, and auroral precipitation with relativistic electron fluxes in all three bands. The correlation for the three parameters with the relativistic electron flux was highest at zero-time lag.

6. The three parameters of Joule heating and auroral precipitation are closely related to the relativistic electron flux and follow a similar 24- 
hour modulation. The ring current is also correlated with relativistic electron flux even if the modulation is not visible.

Despite the findings of this study, additional investigation is necessary to acquire a better understanding of the relativistic electron variation characteristics connected with the entire solar cycle 23. The electron radiation belts are extremely dynamic regions, continually replenished by acceleration and transport of particles. It is important to distinguish regular and climatological variations of the radiation flux from individual responses since they establish the baselines from which fluctuations and large-scale disturbances can be defined and modeled. Further research into the nonlinear impacts of the interaction and the effects of radial transport and local acceleration is still required, necessitating more complex models and providing new insights into the long-term and regular variability.

\section{ACKNOWLEDGEMENTS}

The data of AE, SYM-H, and AL used in this work were obtained from the OMNI website: https://omniweb.gsfc.nasa.gov/form/omni_min.htm 1 and the GOES relativistic electron flux data are provided by NOAA: https://www.ngdc.noaa.gov/ stp/satellite/goes/dataaccess.html. This work is facilitated and supported by the National Science and Research Society (NSRS).

\section{REFERENCES}

[1] Thapa, T.; Adhikari, B.; Baruwal, P. and Pudasainee, K. Variability of Relativistic Electron Flux (E > $2 \mathrm{MeV}$ ) during Geo-Magnetically Quiet and Disturbed days: A Case Study (2020).

[2] Mishra, R. K.; Adhikari, B.; Chapagain, N. P.; Baral, R.; Das, P. K.; Klausner, V. and Sharma, M. Variation on Solar Wind Parameters and Total Electron Content Over Middle-to Low-Latitude Regions During Intense Geomagnetic Storms. Radio Science, 55(11): e2020RS007129 (2020).

[3] Wrenn, G. L. Conclusive evidence for internal dielectric charging anomalies on geosynchronous communications spacecraft. J. Spacecraft Rockets, 32: 514-520 (1995).

[4] Horne, R. B.; Glauert, S. A.; Meredith, N. P.; Boscher, D.; Maget, V.; Heynderickx, D. and Pitchford, D. Space weather impacts on satellites and forecasting the Earth's electron radiation belts with SPACECAST. Space Weather, 11: 169-186 (2013).

[5] Millan, R. M. \& Thorne, R. M. Review of radiation belt relativistic electron losses. Journal of Atmospheric and Solar-Terrestrial Physics, 69(3): 362-377 (2007).

[6] Schiller, Q.; Li, X.; Blum, L.; Tu, W.; Turner, D. L. and Blake, J. B. A nonstorm time enhancement of relativistic electrons in the outer radiation belt. Geophysical Research Letters, 41(1): 7-12 (2014).

[7] Miyoshi, Y.; Kataoka, R.; Kasahara, Y.; Kumamoto, A.; Nagai, T. and Thomsen, M. F. High-speed solar wind with southward interplanetary magnetic field causes relativistic electron flux enhancement of the outer radiation belt via enhanced condition of whistler waves. Geophysical Research Letters, 40(17): 4520-4525 (2013).

[8] Li, W. and Hudson, M. K. Earth's Van Allen radiation belts: From discovery to the Van Allen Probes era. Journal of Geophysical Research: Space Physics, 124(11): 8319-8351 (2019).

[9] Paulikas, G. A. and Blake, J.B. Effects of the solar wind on magnetospheric dynamics: Energetic electrons at the synchronous orbit. Geophysical Monograph Series, 21: 180-202 (1979).

[10] Blake, J. B.; Baker, D. N.; Turner, N.; Ogilvie, K. W. and Lepping, R. P. Correlation of changes in the outer-zone relativistic-electron population with the upstream solar wind and magnetic field measurements. Geophysical Research Letters, 24(8): 927-929 (1997).

[11] Baker, D.; Li, X.; Blake, J. and Kanekal, S. Strong electron acceleration in the Earth's magnetosphere. Advances in Space Research, 21(4): 609-613 (1998).

[12] Moldwin, M. An introduction to space weather. Cambridge University Press (2008).

[13] Li, X.; Baker, D. N.; Kanekal, S. G.; Looper, M. and Temerin, M. Long term measurements of radiation belts by SAMPEX and their variations. Geophys. Res. Lett., 28: 3827-3830 (2001).

[14] Kanekal, S. G.; Baker, D. N. and McPherron, R. L. On the seasonal dependence of relativistic electron fluxes. Ann. Geophys., 28:1101-1106 (2010).

[15] Russell, C. T. and McPherron, R. L. Semi-annual variation of geomagnetic activity. J. Geophys. Res.-Space, 78: 92-108 (1973).

[16] Cortie, A. L. Sun-spots and Terrestrial Magnetic Phenomena,1898-1911: the Cause of the Annual Variation in Magnetic Disturbances. Mon. Not. R. Astron. Soc., 73: 52-60 (1912).

[17] Boller, B. R. and Stolov, H. L. Kelvin-Helmholtz instability and the semi-annual variation of geomagnetic activity, J.Geophys. Res.-Space, 75: 6073-6084 (1970).

[18] Baker, D. N.; Kanekal, S. G.; Pulkkinen, T. I. and Blake, J. B. Equinoctial and solstitial averages of 
magnetospheric relativistic electrons: A strong semi-annual modulation. Geophys. Res. Lett., 26: 3193-3196 (1999).

[19] Chapman, S. Earth storms: Retrospect and prospect. J. Phys. Soc. Japan, 17 (Suppl. A-I): 616 (1962).

[20] Williams, D. J. Ring current and radiation belts. Reviews of Geophysics, 25(3); 570-578 (1987).

21] Daglis, I. A.; Thorne, R. M.; Baumjohann, W. and Orsini, S. The terrestrial ring current: Origin, formation, and decay. Rev. Geophys, 37: 407-438 (1999a).

[22] Daglis, I. A. Ring current dynamics. Space science reviews, 124(1-4): 183-202 (2006).

[23] Hanslmeier, A. The sun and space weather (2. ed). Springer (2007).

[24] Bothmer, V. and Daglis, I. A. Space weather: Physics and effects. Springer-Verlag (2007).

[25] National Centers for Environmental Information. Available Online: www.ngdc.noaa.gov/ngdcinfo/ aboutngdc.html (accessed on 20 April 2021).

[26] Østgaard, N.; Vondrak, R. R.; Gjerloev, J. W. and Germany, G. A. A relation between the energy deposition by electron precipitation and geomagnetic indices during substorms. Journal of Geophysical. Research, 107, in press (2002a).

[27] Østgaard, N. Energy analysis of substorms based on remote sensing techniques, solar wind measurements, and geomagnetic indices. Journal of Geophysical Research, 107(A9): (2002b).

[28] Usoro, A. E. Some basic properties of crosscorrelation functions of $\mathrm{n}$-dimensional vector time series. Journal of Statistical and Econometric Methods, 4(1): 63-71 (2015).

[29] Adhikari, B.; Dahal, S. and Chapagain, N. P. Study of field-aligned current (FAC), interplanetary electric field component (Ey), interplanetary magnetic field component $(\mathrm{Bz})$ and northward (x) and eastward (y) components of the geomagnetic field during super substorm. Earth and Space Science, 4(5): 257-274 (2017b).

[30] Silwal, A.; Gautam, S. P.; Poudel, P.; Karki, M.; Adhikari, B.; Chapagain, N. P.; ... \& MigoyaOrue, Y. GPS Observations of Ionospheric TEC Variations during the 15th Jan 2010 and 21st June 2020 Solar Eclipse. Radio Science, 56: e2020RS007215 (2021).

[31] Katz, R. W. Use of cross-correlations in the search for teleconnections. Journal of Climatology, 8(3): 241-253 (1988).

[32] Mishra, R. K.; Silwal, A.; Baral, R.; Adhikari, B.; Braga, C. R.; Gautam, S. P.; ... and Migoya-Orue, Y. Singularity Detection on Forbush Decrease at High Latitude Stations During Geomagnetic Disturbances. Preprint (2021).
[33] Adhikari, B.; Dahal, S.; Sapkota, N.; Baruwal, P.; Bhattarai, B. and Chapagain, N. P. Field Aligned Current and Polar Cap Potential and Geomagnetic Disturbances: A Review of Cross-Correlation Analysis. Earth and Space Science, 5: 440-455 (2018).

[34] Poudel, P.; Parajuli, N.; Gautam, A.; Sapkota, D.; Adhikari, H.; Adhikari, B.; Silwal, A.; Gautam, S.; Karki, M. and Mishra, R. Wavelet and CrossCorrelation Analysis of Relativistic Electron Flux with Sunspot Number, Solar Flux, and Solar Wind Parameters. Journal of Nepal Physical Society, 6(2): 104-112 (2020).

[35] Li, L. Y.; Yang, S. S.; Cao, J. B.; Yu, J.; Luo, X. Y. and Blake, J. B. Effects of solar wind plasma flow and interplanetary magnetic field on the spatial structure of Earth's radiation belts. Journal of Geophysical Research: Space Physics, 124(12): 10332-10344 (2019).

[36] Robinson, R. M. and Zanetti, L. J. Auroral energy flux and joule heating derived from global maps of field aligned currents. Geophysical Research Letters, 48(7): e91527 (2021).

[37] Le, G.; Russell, C. T. and Takahashi, K. Morphology of the ring current derived from magnetic field observations. Annales Geophysicae, 22(4): 1267-1295 (2004).

[38] Yeeram, T. Solar activity phase dependence of the magnetospheric processes and relativistic electron flux at geostationary orbit. Astrophysics and Space Science, 365: 86 (2020).

[39] Adhikari, B.; Adhikari, R.; Chapagain, N. P.; Sapkota, N.; Dahal, S. and Pandit, D. Daily, Seasonal and Monthly Variation of Middle-low latitudes Magnetic Field during Low Solar Activity. Discovery, 53(255): 181-190 (2017). 